\section{P2-S7.06 FACTORIAL VALIDITY AND INTERNAL CONSISTENCY OF THE SCALE OF ATTITUDE TOWARDS PEOPLE LIVING WITH HIV AND AIDS}

doi:10.1136/sextrans-2011-050108.373

${ }^{1} \mathrm{M}$ Beaulieu, ${ }^{2} \mathrm{~A}$ Adrien, ${ }^{1} \mathrm{~L}$ Potvin, ${ }^{1} \mathrm{C}$ Dassa. ${ }^{1}$ Université de Montréal, Montréal, Canada; ${ }^{2}$ Direction de Santé Publique de Montréal, Montréal, Canada

Background The objective of the study was to investigate the factorial validity and internal consistency of the Scale of Attitude towards People Living with HIV and AIDS (SAPLWHA).

Methods Between 15 March and 2 April 2010, a telephone survey was conducted among a representative sample of 1500 English and French speaking Quebecers between the ages of 15-64 years. The factorial validity of the scale was investigated in two steps: first, with exploratory factor analyses (EFA) (Principal Axis Factoring with oblique rotation) and second, with confirmatory factor analyses (CFA) (Weighted Least Squares estimation with polychoric correlations). The original sample was randomly separated into two subsamples and independently used for the EFA and CFA. Different indicators were used to assess the factorial structure.

Results The best EFA model had a simple structure of seven moderately correlated factors: (F1): Fear of being infected; (F2): Personal contact; (F3): Prejudice, perceptions of risk groups; (F4): Liberalism; (F5): Social Support; (F6): Right to be informed / HIV confidentiality; (F7): Support to the criminalisation of disease transmission. This factor structure explained $44 \%$ of the variance which represents $76 \%$ of the total variance when the data are reduced to 7 dimensions. The reliability analysis showed a high internal consistency for the total scale (0.89), adequate values for all factors except F6 (0.70 to 0.79), and a moderate value for F6 (0.59). Although the CFA $\chi^{2}$ test was not significant, fit indexes indicated that the seven factor model fits the data (Root Mean Square Error Of Approximation $=0.0396$; Comparative Fit Index $=0.950$; NonNormed Fit Index=0.942; Goodness-of-Fit Index=0.981).

Conclusions Results suggest that the SAPLWHA is a measure that adequately documents the current attitudes towards PLWHA. Factorial scores derived from this scale will allow external validity assessment as well as provide useful information for future awareness and compassion campaigns designed for the general population.

\section{P2-S7.07 ADHERENCE AND DIFFICULTIES WITH ANTIRETROVIRAL MEDICATION IN AN AUSTRALIAN SAMPLE OF PEOPLE LIVING WITH HIV}

doi:10.1136/sextrans-2011-050108.374

A Lyons, J Grierson, R Koelmeye, A Smith, M Pitts. La Trobe University Melbourne, Australia

Background While combination antiretroviral therapy (cART) has revolutionised the course of HIV infection, a high level of adherence to CART is required to achieve the full mortality benefit. While a number of studies have investigated factors associated with non-adherence to cART, there has been considerable variability in the findings. In this study we set out to determine which personal, socioeconomic, treatment-related and disease-related factors are independently associated with adherence to ARV in an Australian sample of PLWH.

Methods Using data from the HIV futures six survey, an anonymous cross-sectional survey of PLWH conducted in 2009, we conducted bivariate and multivariate analyses to assess the association of factors with self-reported difficulty taking ARV. Chi-square and $t$ tests were used for bivariate analysis. A two-step logistic regression modelling procedure based on backwards stepwise regression was used for multivariate analysis. Factors that demonstrated a signifi- cant association at $\alpha=0.2$ in bivariate analysis were included in multivariate analysis.

Results We identified 75 variables within our data that were likely to be associated with difficulty taking ARV. 45 met multivariate analysis inclusion criteria. Factors found to be independently associated at $a=0.05$ : younger age, alcohol and party drug use, diagnosis of mental health condition, poor or fair self-reported health, living in a regional centre, taking more than 1 ARV dose/day, experiencing adverse physical events in the last 12 months, health service discrimination in the last 2 years, using a nucleotide analogue reverse transcriptase inhibitor or protease inhibitor and specific attitudes: not believing in the benefits of ARV, concern about medication efficacy in the future and thinking that ARV tablets were an unwanted reminder of having HIV.

Conclusion The multitude of factors found to be independently associated with difficulty taking ARV reaffirms the dynamic nature of adherence behaviour and the ongoing importance of addressing adherence behaviour in the clinical management of PLWH.

\section{P2-S7.08 PSYCHOSOCIAL SUPPORT AND THERAPEUTIC ADHERENCE OF HIV POSITIVE PREGNANT WOMEN AT HIV TREATMENT HOSPITAL OF SURU LÉRÉ IN COTONOU (BENIN)}

doi:10.1136/sextrans-2011-050108.375

K Adolphe Codjo, S S Mistourath. University of Abomey Calavi Benin, Cotonou, Benin

Context The sociocultural contexts and social environnement of people living with HIV and especially of HIV positive pregnant women are poorly documented and often not integrated with global care of HIV in Benin. This study describes and analyzes the interactions between healthcare professionals and HIV positive pregnant women to better understand the challenges of the medical staff to guide patients in their social environment.

Method Data were collected through documentary analysis, direct observation, semi structured interview, Focus Group with healthcare professionals, HIV positive pregnant women, their family and social environment. The study focused on 130 respondents. Analysis was done with the triangulation method and content analysis.

Result Academic training, professional experiences with patients direct the activities and practices of nursing staff. Therapeutic Relations determine the management of confidentiality. For $90 \%$ of HIV positive pregnant women interviewed, it is within the system or hospital or medical device (with the health personnel) that they find psychosocial support to be safe from the stigmatisation. It results in low therapeutic adherence and barriers to the Preventing Mother to child transmission of HIV.

Conclusion The stigmatisation of people with the AIDS virus remains present in society and influenced the Behaviours of Patients. The healthcare professionals-patient relationships built on mutual trust and focused primarily on the medical device to the detriment of the social environment of HIV positive pregnant women not guarantee continuity of care and support for people living with HIV.

\section{Social and behavioural aspects of prevention poster session 8: Technology and Media P2-S8.01 ABSTRACT WITHDRAWN}

P2-S8.02 ABSTRACT WITHDRAWN 